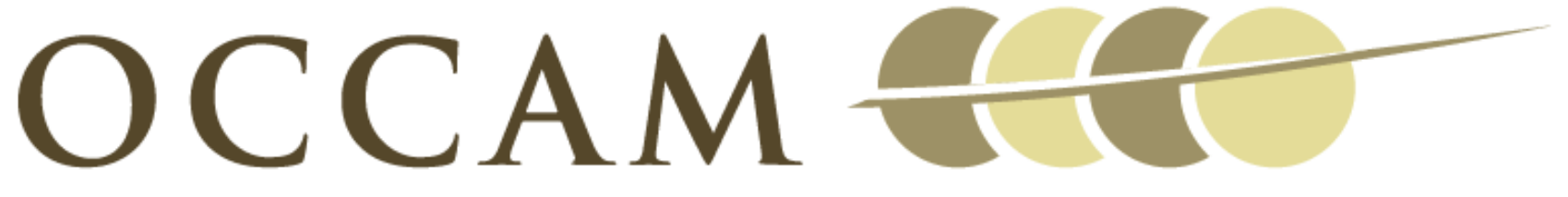

OXFORD CENTRE FOR COLLABORATIVE APPLIED MATHEMATICS

Report Number 10/04

Random intermittent search and the tug-of-war model of motor-driven transport

by

Jay Newby, Paul C. Bressloff

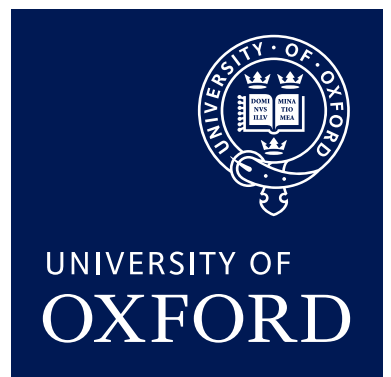

Oxford Centre for Collaborative Applied Mathematics Mathematical Institute 24 - 29 St Giles'

Oxford

OX1 3LB

England 



\title{
Random intermittent search and the tug-of-war model of motor-driven transport
}

\author{
Jay Newby and Paul C Bressloff \\ Department of Mathematics, University of Utah, Salt Lake City, Utah 84112, \\ USA \\ Mathematical Institute, University of Oxford, 24-29 St. Giles', Oxford OX1 \\ $3 \mathrm{LB}, \mathrm{UK}$ \\ E-mail: bressloff@math.utah.edu
}

\begin{abstract}
We formulate the "tug-of-war" model of microtubule cargo transport by multiple molecular motors as an intermittent random search for a hidden target. A motor-complex consisting of multiple molecular motors with opposing directional preference is modeled using a discrete Markov process. The motors randomly pull each other off of the microtubule so that the state of the motorcomplex is determined by the number of bound motors. The tug-of-war model prescribes the state transition rates and corresponding cargo velocities in terms of experimentally measured physical parameters. We add space to the resulting Chapman-Kolmogorov (CK) equation so that we can consider delivery of the cargo to a hidden target somewhere on the microtubule track. Using a quasisteady state (QSS) reduction technique we calculate analytical approximations of the mean first passage time (MFPT) to find the target. We show that there exists an optimal adenosine triphosphate (ATP) concentration that minimizes the MFPT for two different cases: (i) the motor complex is composed of equal numbers of kinesin motors bound to two different microtubules (symmetric tugof-war model), and (ii) the motor complex is composed of different numbers of kinesin and dynein motors bound to a single microtubule (asymmetric tug-of-war model).
\end{abstract}




\section{Introduction}

Random search strategies are used throughout nature as a means of efficiently searching large areas for one or more targets of unknown location. Examples include animals foraging for food or shelter $[1,2,3]$, proteins searching for particular sites on DNA $[4,5]$, and biochemical reaction kinetics [6]. Recently, there has been a great deal of interest in a particular class of search strategy known as random intermittent search, in which a searcher randomly switches between a slow search phase and a faster non-search phase $[7,8,9,10,11]$. At the macroscopic scale, intermittent motion has been observed in a variety of animal species during exploratory behavior [12]. One striking example is given by the nematode C.elegans, which alternates between a fast displacement along a straight trajectory (roaming) and a much slower displacement along a more sinuous trajectory (dwelling) [13]. During the slow phase, the worm's head, bearing most of its sensory organs, moves and touches the surface nearby suggesting that this is a search phase. Intermittent motion also occurs at the microscopic level of reaction kinetics within biological cells. For example, diffusing molecules within the cellular environment intermittently bind and unbind to molecular motors that execute ballistic transport along cytoskeletal filaments powered by adenosine triphosphate (ATP) hydrolysis; reaction with a target molecule can only occur during the free diffusive phase $[6,14]$. Experimental observations of the dynamic behavior of molecular motor-cargo complexes transported along microtubules reveal intermittent behavior with constant velocity movement in both directions along the microtubule, interrupted by brief pauses or fast oscillatory movements that may correspond to localization at specific targets $[17,18,19,20,21]$. Motor-driven transport mechanisms are important in cellular function for two reasons. First, spatially extended cells such as neurons cannot distribute resources from the cell body to extended cellular compartments such as axons and dendrites via Brownian motion alone as the distances are too great. Second, many cells require targeting of resources to specific sub-cellular regions necessitating a method of directed transport. The latter also implies that cellular mechanisms must be employed for sorting and localizing motor-driven cargo to their intended destination. Many of the mechanisms that localize cargo are known to be associated with the target. For example, several proteins are known to cause the motor to detach from its cargo so that local concentrations of these proteins around a target may facilitate its localization [22]. In this paper we explore the possibility that the random intermittent motion of a motor-cargo complex may itself facilitate the search and delivery process.

There is growing evidence that the molecular motor-based bidirectional transport of cargo along microtubules is a result of the combined action of multiple motors attached to the cargo; the current velocity state is determined by the subset of motors currently bound to a microtubule track [23]. Microtubules are polarized filaments with biophysically distinct $(+)$ and $(-)$ ends, and this polarity determines the preferred direction in which an individual molecular motor moves. For example, kinesin moves towards the $(+)$ end whereas dynein moves towards the $(-)$ end. In the axons and dendrites of neurons that are located far from the cell body one finds that microtubule filaments all have the same polarity, with the $(+)$ end oriented away from the cell body. This suggests a model of bidirectional transport in which kinesin and dynein motors transport a cargo in opposite directions along a single track. On the other hand, dendritic microtubules located close to the cell body tend to have mixed polarities [24], suggesting a model in which motors of the same directional 
preference are distributed among two parallel microtubules of opposite polarity. In both of the above scenarios, there has to be some mechanism for coordinating the action of the various motors as part of a larger motor complex. One possibility is that the motors interact through a tug-of-war competition, where individual motors influence each other through the force they exert on the cargo [23,25]. When a force is exerted on a motor opposite to its preferred direction, it is more likely to detach from its microtubule. Ultimately the motion of the cargo is determined by the random attachments and force-dependent detachments from the microtubule of each motor in the motor complex. A corresponding biophysical model of motor competition has also been developed, in which the transitions between the different internal motor states are described in terms of a discrete Markov process [25,26]. However, the location of the motor complex along a filament track is not considered.

In this paper we present a model of random intermittent search of active microtubular transport within cells, based on the tug-of-war model of motor-driven cargo transport. The stochastic search process is modeled in terms of a differential Chapman-Kolmogorov (CK) equation that keeps track of both the spatial location and the internal states of the motor-complex, with the latter identified with the number of kinesin and/or dynein motors attached to the microtubule. In order to make the analysis of the CK equation tractable, we carry out a quasi-steady state (QSS) or adiabatic reduction of the full model using a projection method [27,28], extending our previous analysis of a 3-state intermittent search model [16]. The QSS reduction is valid when the transition rates among the internal states of the motor-complex are fast compared to the rate at which an individual motor travels a fundamental distance along the track, which is taken to be the size of the target. Under this approximation, the probability distribution over internal states rapidly converges to the stationary distribution of the associated "tug-of-war" master equation studied by Muller et. al. $[25,26]$; the projected probability density in space then evolves according to a scalar Fokker-Planck (FP) equation with an additional inhomogeneous decay term that takes into account absorption by a target. Using the reduced FP equation, we compute the mean first passage time (MFPT) to target absorption and show that there exists an optimal level of ATP concentration, [ATP], at which the MFPT is minimized. This suggest that $[\mathrm{ATP}]$ could act as a control signal for optimizing the search process.

The structure of the paper is as follows. In section 2 we review the basic tug-ofwar model $[25,26]$ and show how to extend it to include the effects of changes in ATP concentration. We then reformulate the tug-of-war model as a random intermittent search for a hidden target along a one-dimensional filament track. We carry out the QSS reduction of the full model in section 3, and use this to derive an analytically tractable expression for the MFPT to find the target. This allows us to explore both analytically and numerically how $[\mathrm{ATP}]$ affects cargo delivery under various circumstances. First, we explore how $[$ ATP $]$ affects the mean delivery time when the intermittent motion is unbiased (section 4.1). In order to make the motion of the motor complex unbiased, we assume that two sets of identical kinesin motors move in opposite directions which corresponds to the symmetric tug-of-war model [26]. We then extend the analysis to the case of biased or directed intermittent search corresponding to an asymmetric tug-of-war model, in which there are different numbers of forward moving kinesin motors and backward moving dynein motors moving along a single track (section 4.2). 


\section{Tug-of-war model of microtubule cargo transport}

Suppose that a certain vesicular cargo is transported along a one-dimensional track via $N_{+}$right-moving (anterograde) motors and $N_{-}$left-moving (retrograde motors). At a given time $t$, the internal state of the cargo-motor complex is fully characterized by the numbers $n_{+}$and $n_{-}$of anterograde and retrograde motors that are bound to a microtubule and thus actively pulling on the cargo. We assume that over the timescales of interest all motors are permanently bound to the cargo so that $0 \leq n_{ \pm} \leq N_{ \pm}$. The binding and unbinding rates and the cargo velocities are obtained from the tug-ofwar model [25]. This assumes that the motors act independently other than exerting a load on motors with the opposite directional preference. Thus the properties of the motor complex can be determined from the corresponding properties of the individual motors together with a specification of the effective load on each motor. There are two distinct mechanisms whereby such bidirectional transport could be implemented [26]. First, the track could consist of a single polarized microtubule filament (or a chain of such filaments) on which up to $N_{+}$kinesin motors and $N_{-}$dynein motors can attach, see Fig. 1. Since individual kinesin and dynein motors have different biophysical properties, with the former tending to exert more force on a load, it follows that even when $N_{+}=N_{-}$the motion will be biased in the anterograde direction. Hence, this version is referred to as an asymmetric tug-of-war model. Alternatively, the track could consist of two parallel microtubule filaments of opposite polarity such that $N_{+}$ kinesin motors can attach to one filament and $N_{-}$to the other, see Fig. 2 . In the latter case, if $N_{+}=N_{-}$then the resulting bidirectional transport is unbiased and we have a symmetric tug-of-war model.

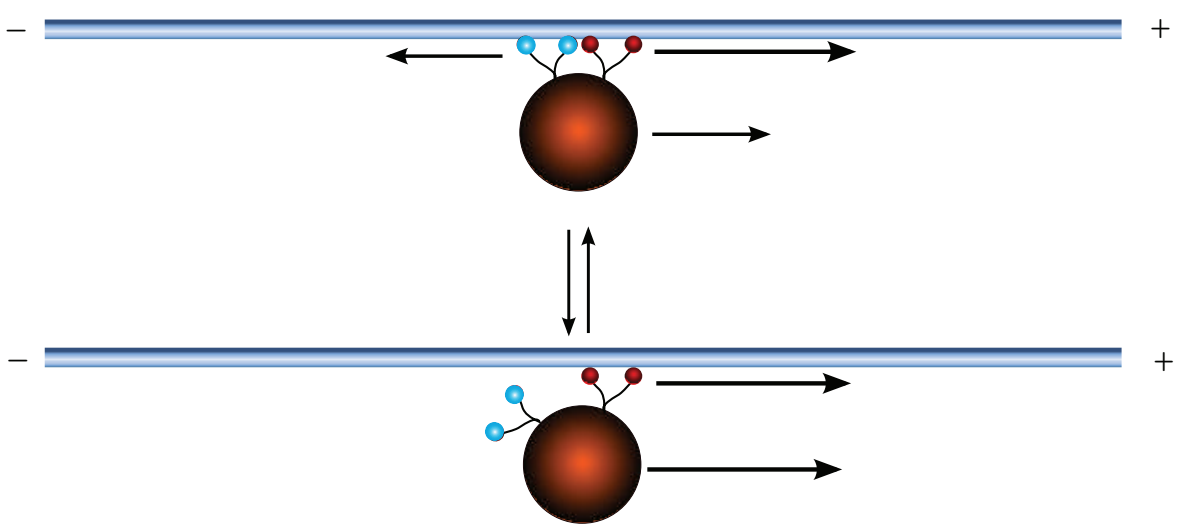

Figure 1. Schematic diagram of an asymmetric tug-of-war model. Two kinesin and two dynein motors transport a cargo in opposite directions along a single polarized microtubule track. Transitions between two possible motor states are shown.

When bound to a microtubule, each motor has a load-dependent velocity

$$
v(F)= \begin{cases}v_{f}\left(1-F / F_{s}\right) & \text { for } F \leq F_{s} \\ v_{b}\left(1-F / F_{s}\right) & \text { for } F \geq F_{s}\end{cases}
$$

where $F$ is the applied force, $F_{s}$ is the stall force satisfying $v\left(F_{s}\right)=0, v_{f}$ is the forward motor velocity in the absence of an applied force in the preferred direction of the particular motor, and $v_{b}$ is the backward motor velocity when the applied force 


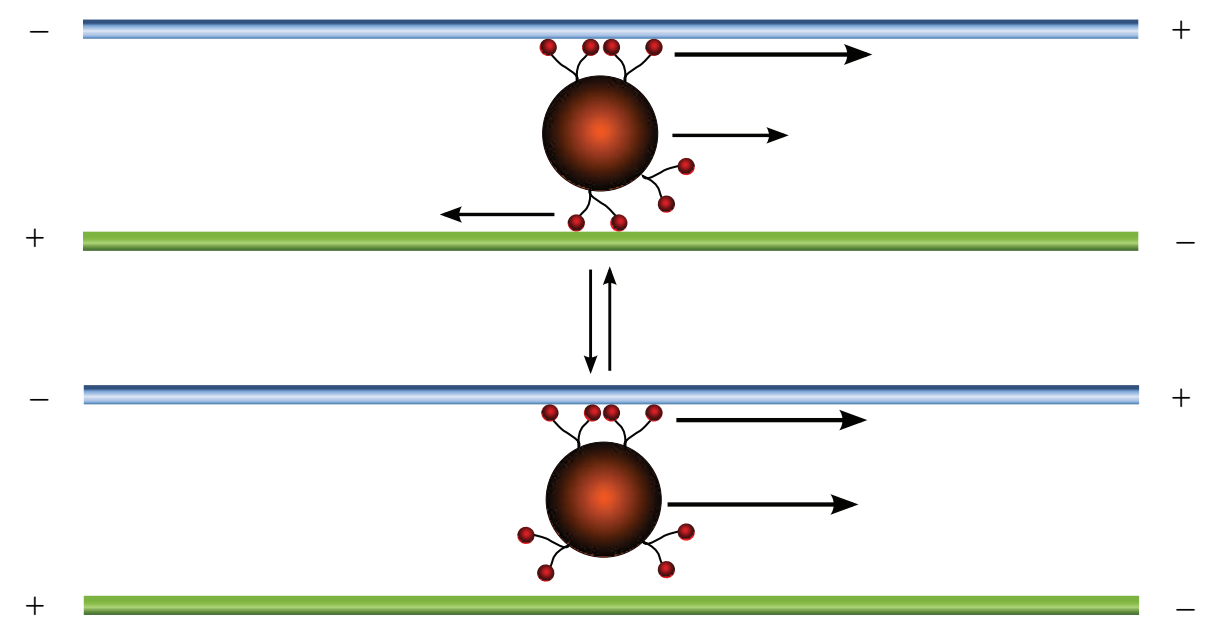

Figure 2. Schematic diagram of a symmetric tug-of-war model. Pairs of motors of the same directional preference are distributed among two parallel microtubules of opposite polarity. Transitions between two possible motor states are shown.

exceeds the stall force. The unbinding rate has been found to be approximately an exponential function of the applied force

$$
\gamma(F)=\gamma_{0} \mathrm{e}^{\frac{F}{F_{d}}},
$$

where $F_{d}$ is the experimentally measured force scale on which unbinding occurs. On the other hand, the binding rate is taken to be independent of load:

$$
\pi(F)=\pi_{0} .
$$

Let $F_{c}$ denote the net load on the set of anterograde motors, which is taken to be positive when pointing in the retrograde direction. It follows that a single anterograde motor feels the force $F_{c} / n_{-}$, whereas a single retrograde motor feels the opposing force $-F_{c} / n_{+}$. Equations (2.2) and (2.3) imply that the binding and unbinding rates for the anterograde and retrograde motors are

$$
\begin{aligned}
& \gamma_{ \pm}\left(n_{+}, n_{-}\right)=n_{ \pm} \gamma_{0 \pm}\left(F_{c} / n_{ \pm}\right) \\
& \pi_{ \pm}\left(n_{ \pm}\right)=\left(N_{ \pm}-n_{ \pm}\right) \pi_{0 \pm} .
\end{aligned}
$$

The cargo force $F_{c}$ is determined by the condition that all the motors move with the same cargo velocity $v_{c}$. Suppose that $N_{+} \geq N_{-}$so that the net motion is in the anterograde direction, which is taken to be positive. In this case we have that the forward motors are stronger than the backward motors so that $n_{+} F_{s+}>n_{-} F_{s-}$. Equation (2.1) implies that

$$
v_{c}=v_{f+}\left(1-F_{c} /\left(n_{+} F_{s+}\right)\right)=-v_{b-}\left(1-F_{c} /\left(n_{-} F_{s-}\right)\right) .
$$

We thus obtain a unique solution for the load $F_{c}$ and cargo velocity $v_{c}$ :

$$
F_{c}\left(n_{+}, n_{-}\right)=\left(\mathcal{F} n_{+} F_{s+}+(1-\mathcal{F}) n_{-} F_{s-}\right),
$$

where

$$
\mathcal{F}=\frac{n_{-} F_{s-} v_{f-}}{n_{-} F_{s-} v_{f-}+n_{+} F_{s+} v_{b-}}
$$


and

$$
v_{c}\left(n_{+}, n_{-}\right)=\frac{n_{+} F_{s+}-n_{-} F_{s-}}{n_{+} F_{s+} / v_{f+}+n_{-} F_{s-} / v_{b-}} .
$$

The corresponding formulas for the case where the backward motors are stronger so that $n_{+} F_{s+}<n_{-} F_{s-}$ are found by interchanging $v_{f}$ and $v_{b}$.

\subsection{ATP dependence}

In the original tug-of-war analysis [25], the motor exhibited three different modes of behavior. In the first mode labeled (0) the motor complex spends most of its time in states with approximately zero velocity. The second mode labeled $(-0+)$ is characterized by fast backward and forward movement interrupted by pauses of no motion, much like intermittent search behavior. Finally, in mode $(-+)$ the cargo alternates between fast backward and forward movement. The transitions between these modes of behavior depend on motor strength which primarily depends upon the stall force. A convenient experimental method of changing motor strength is to vary the level of ATP available to the motor complex. At low $[\mathrm{ATP}]$ the motor has little fuel and is weaker, resulting in (0) mode behavior, then as [ATP] increases and more fuel is available $(-0+)$ behavior is seen until the stall force saturates at high values of $[$ ATP] where $(-+)$ mode behavior takes over. Thus, $[\mathrm{ATP}]$ provides a single control parameter that tunes the level of intermittent behavior exhibited by a motor complex. Others have developed models of the [ATP] and force dependent motor parameters that closely match experiments for both kinesin [29,30] and dynein [31,32]. Based on these studies we take the forward velocity of a single motor under zero to have the Michaelis-Menten form

$$
v_{f \pm}([\mathrm{ATP}])=\frac{v_{f \pm}^{\max }[\mathrm{ATP}]}{[\mathrm{ATP}]+K_{m \pm}^{0}}
$$

The backward velocity is small $\left(v_{b \pm}= \pm 0.006 \mu \mathrm{m} / \mathrm{s}\right)$ so that we can ignore its [ATP] dependence. The unbinding rate of a single motor under zero load can be determined using the $[\mathrm{ATP}]$ dependent average run length $L_{\rho \pm}([\mathrm{ATP}])$. The mean time to detach from the microtubule is $v_{f \pm}([\mathrm{ATP}]) / L_{\rho \pm}([\mathrm{ATP}])$ so that

$$
\gamma_{0 \pm}([\mathrm{ATP}])=\frac{v_{f \pm}^{\max }\left([\mathrm{ATP}]+K_{p \pm}\right)}{L_{\rho \pm}^{\max }\left([\mathrm{ATP}]+K_{m \pm}^{0}\right)}
$$

The binding rates are determined by the time necessary for an unbound motor to diffuse within range of the microtubule and bind to it, which is assumed to be independent of both load and $[\mathrm{ATP}]$. Finally, the $[\mathrm{ATP}]$ dependent stall force is given by

$$
F_{s \pm}([\mathrm{ATP}])=F_{s \pm}^{0}+\frac{\left(F_{s \pm}^{\max }-F_{s \pm}^{0}\right)[\mathrm{ATP}]}{K_{s \pm}+[\mathrm{ATP}]} .
$$

In the asymmetric model we take the parameters of the anterograde (retrograde) motors to correspond to those obtained experimentally for kinesin (dynein), whereas in the symmetric model both sets of parameters are identified with those of kinesin. A list of parameter values consistent with experiments are found in Table 1. 
Table 1. Tug-of-war parameter values

\begin{tabular}{lll}
\hline Parameter & Kinesin & Dynein \\
\hline$v_{f}^{\max }(\mu \mathrm{m} / \mathrm{s})$ & 1 & 0.7 \\
$K_{m}^{0}(\mu M)$ & 79 & 38 \\
$L^{\max }(\mu \mathrm{m})$ & 0.86 & 1.5 \\
$K_{p}(\mu M)$ & 3.13 & 1.5 \\
$F_{s}^{0}(p N)$ & 0.22 & 5.5 \\
$F_{s}^{\max }(p N)$ & 1.24 & 8 \\
$K_{s}(p N)$ & 100 & 480 \\
$F_{d}(p N)$ & 6 & 6 \\
$\pi_{0}\left(\mathrm{~s}^{-1}\right)$ & 5 & 1.6 \\
$v_{b}(\mu \mathrm{m} / \mathrm{s})$ & 0.006 & 0.006 \\
\hline
\end{tabular}

\subsection{Generalized intermittent search model}

The tug-of-war model [25] considers the stochastic dynamics associated with transitions between different internal states $\left(n_{+}, n_{-}\right)$of the motor-cargo complex, without specifying the spatial position of the cargo along a one-dimensional track. This defines a Markov process with a corresponding master equation for the time evolution of the probability distribution $p\left(n_{+}, n_{-}, t\right)$. In order to apply the model to the problem of random intermittent search, it is necessary to construct a differential CK equation for the probability density $p\left(n_{+}, n_{-}, x, t\right)$ that the cargo is in the internal state $\left(n_{+}, n_{-}\right)$and has position $x$ at time $t$. The intermittent search problem for the tug-of-war model is then formulated as follows, see Fig. 3. Suppose a particle is moving along a one dimensional track of length $L$ having started at some location $0 \leq y \leq L$. A target is introduced somewhere within the track at location $x=X$ so that if the particle is within a distance $l$ of the target and in a searching state it can locate the target at a rate $k_{0}$. For the tug-of-war model, we assume that the system is in a slowly moving search state, i.e. able to detect the target, if the number of anterograde and retrograde motors are equal, $n_{+}=n_{-}$, whereas it is in a fast moving non-search state when $n_{+} \neq n_{-}$. Since kinesin and dynein have different biophysical properties, the velocity $v_{c}(n, n)$ in states with equal numbers of opposing motors engaged will be small but not identically zero for the asymmetric tug-of-war model, whereas in the symmetric case $v(n, n)=0$.

Previous studies of random intermittent search typically consider two basic types of search state [10], a static (or slow ballistic) state and a slowly diffusing state. In this paper we focus on the static (or slow ballistic) case since free diffusion of a cargo during motor-transport can only occur when all motors are detached from the microtubule and often the cargo is many orders of magnitude larger than the motor complex, making any diffusive motion negligible on the space scale of the microtubule track. However, recent studies of motor transport have shown that myosin, a molecular motor associated with actin filaments, can bind to and diffuse along microtubules $[33,34,35]$. These myosin tethers have also been incorporated into the tug-of-war model [36], and other similar transport models [37]. For the sake of general applicability to tug-of-war models that also take into account the effects of a diffusing tether, we include diffusion in the formulation of the model and the QSS reduction.

In order to write down the CK equation, it is covenient to introduce the label 


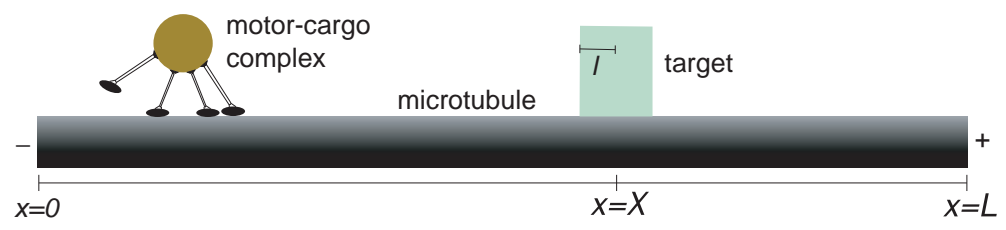

Figure 3. Diagram depicting the tug-of-war model of random intermittent search for a hidden target on a one-dimensional track of length $L$. (For the sake illustration we show the asymmetric case). Two populations of motors which move in opposite directions bind and unbind to the microtubule. When equal numbers of each type of motor are bound, the cargo is in a searching state and can locate the target centered at $x=X$ and of width $2 l$ provided that it is within range.

$i\left(n_{+}, n_{-}\right)=\left(N_{+}+1\right) n_{-}+\left(n_{+}+1\right)$ and set $p\left(n_{+}, n_{-}, x, t\right)=p_{i\left(n_{+}, n_{-}\right)}(x, t)$. We then have an $n$-component probability density vector $\boldsymbol{p} \in \mathbb{R}^{n}$ with $n=\left(N_{+}+1\right)\left(N_{-}+1\right)$. The corresponding differential CK equation takes the form [28]

$$
\partial_{t} \boldsymbol{p}=A \boldsymbol{p}-\mathcal{L}(\boldsymbol{p}),
$$

where $A \in \mathbb{R}^{n \times n}$ specifies the transition rates between each of the $n$ internal motor states and the differential operator $\mathcal{L}$ has the structure

$$
\mathcal{L}=\left[\begin{array}{ccccc}
\mathcal{L}_{1} & 0 & \ldots & & 0 \\
0 & \mathcal{L}_{2} & 0 & \ldots & 0 \\
\vdots & & \ddots & & \vdots \\
& & & \mathcal{L}_{n-1} & 0 \\
0 & \cdots & & 0 & \mathcal{L}_{n}
\end{array}\right]
$$

where the scalar operators are given by

$$
\mathcal{L}_{j}=\left[\kappa_{j}(x)+v_{j} \partial_{x}-B_{j} \partial_{x}^{2}\right] .
$$

Here $v_{j}=v_{c}\left(n_{+}, n_{-}\right)$is the velocity of internal state $j=j\left(n_{+}, n_{-}\right), B_{j}$ is the diffusivity, and $\kappa_{j}(x)$ is the rate of target detection at location $x$. Assuming that the target is at an unknown location $X$ and is of half-width $l$, we set $\kappa_{i}(x)=k_{i} \chi(x)$ where $\chi(x)=1$ if $|x-X|<l$ and zero otherwise,

$$
k_{i}=\left\{\begin{array}{cc}
k_{0} & \text { if }\left|v_{i}\right| \leq \bar{v} \\
0 & \text { otherwise }
\end{array}\right.
$$

and $\bar{v}=\max _{n}\{v(n, n)\}$. Thus the searcher can only find the target if it is within range and in a slowly moving search state. The diffusivities are taken to be zero unless all motors are detached from the microtubule. We assume that the motor complex remains in the vicinity of the track due to the action of a tether, say, which diffuses along the track at a rate $D_{0}$ so that

$$
B_{i}=\left\{\begin{array}{cc}
D_{0} & \text { if } i=1 \\
0 & \text { otherwise }
\end{array}\right.
$$

The components $a_{i, j}, i, j=1, \ldots, n$, of the state transition matrix $A$ are given by the corresponding binding/unbinding rates of equations (2.4) and (2.5). That is, setting $i=i\left(n_{+}, n_{-}\right)$, the non-zero off-diagonal terms are

$$
a_{i, j}=\pi_{+}\left(n_{+}-1\right) \text { for } j=i\left(n_{+}-1, n_{-}\right)
$$




$$
\begin{aligned}
& a_{i, j}=\pi_{-}\left(n_{-}-1\right), \text { for } j=i\left(n_{+}, n_{-}-1\right) \\
& a_{i, j}=\gamma_{+}\left(n_{+}+1, F_{c}\right), \text { for } j=i\left(n_{+}+1, n_{-}\right),
\end{aligned}
$$

and

$$
a_{i, j}=\gamma_{-}\left(n_{-}+1, F_{c}\right) \text {, for } j=i\left(n_{+}, n_{-}+1\right) .
$$

The diagonal terms are then given by $a_{i, i}=-\sum_{j \neq i} a_{j, i}$. Finally, it is necessary to specify the initial conditions and boundary conditions at the ends $x=0, L$. In this paper we impose either reflecting or periodic boundary conditions so that there is unit probability that the target will eventually be found. A random search with periodic boundary conditions can be interpreted as a search in a domain with periodically distributed targets of spacing $L$, in which the identity of the first target to be detected is unimportant.

Following previous studies of random intermittent search [10], we are interested in determining the efficiency of the search process by calculating the MFPT to detect a target, which we denote by $\mathcal{T}$, and exploring whether or not $\mathcal{T}$ can be minimized with respect to variations in important biophysical parameters such as the level of ATP concentration. In the case of the tug-of-war model, the MFPT is given by

$$
\mathcal{T}=k_{0} \int_{0}^{\infty} t d t \int_{X-l}^{X+l} d x \sum_{n_{+}, n_{-}} p\left(n_{+}, n_{-}, x, t\right) \delta_{n_{+}, n_{-}} .
$$

For ease of notation we have suppressed the dependence on initial conditions. We will impose an initial condition of the form $p\left(n_{+}, n_{-}, x, 0\right)=\delta(x-y) P_{0}\left(n_{+}, n_{-}\right)$, where $P_{0}$ is the initial distribution over internal states, and either fix $y=0$ (motor complex starts at the left-hand end of the track) or uniformly average over all starting positions $y$. Although it would be possible in principal to calculate $\mathcal{T}$ analytically for the full model, following along similar lines to previous studies of 3 -state models $[10,15]$, the analysis is considerably more involved due to the complexity of the molecular motor model. Therefore, we follow a different approach here by carrying out a QSS reduction of the differential CK equation (2.13) [16]. The reduced model is described by a scalar Fokker-Plank (FP) equation with an extra inhomogeneous decay term accounting for the target-dependent terms in the CK equation. Within this approximation, the MFPT can be expressed in terms of the Laplace transformed solution of the FP equation.

\section{Quasi-steady-state reduction}

Suppose that we fix the units of space and time by setting $l=1$ and $l / v(1,0)=1$, which corresponds to non-dimensionalizing the CK equation (2.13) by performing the rescalings $x \rightarrow x / l$ and $t \rightarrow t v(1,0) / l$. Furthermore, we assume that for the given choice of units, $a_{i j}=\mathcal{O}(1 / \varepsilon)$ whereas $\mathcal{L}_{i j}=\mathcal{O}(1)$ for some small parameter $\varepsilon \ll 1$, and set $A=\varepsilon^{-1} \widehat{A}$. We can then carry out a QSS reduction of the dimensionless CK equation

$$
\partial_{t} \boldsymbol{p}=\frac{1}{\varepsilon} \widehat{A} \boldsymbol{p}-\mathcal{L}(\boldsymbol{p})
$$

following along similar lines to our previous analysis of a three-state model of random intermittent search [16]. It is straightforward to show that the matrix $\widehat{A}$ is irreducible and is conservative so that $\boldsymbol{\psi}=(1,1, \cdots, 1)^{T}$ is in the nullspace of $\widehat{A}^{T}$. Since $\widehat{A}$ 
has one zero eigenvalue it follows from the Perron-Frobenius theorem that all of the remaining eigenvalues have negative real part, and the right nullvector has all real positive entries. Let $\boldsymbol{p}^{s s} \in N(\widehat{A})$ and choose $\boldsymbol{p}^{s s}$ so that $\boldsymbol{\psi}^{T} \boldsymbol{p}^{s s}=1$ so that $\boldsymbol{p}^{s s}$ is the stationary distribution for the tug-of-war process. We may then define the projection operator $\mathcal{P}=\boldsymbol{p}^{s s} \boldsymbol{\psi}^{T}$ and decompose $\boldsymbol{p}$ into two parts:

$$
\boldsymbol{p}=u \boldsymbol{p}^{s s}+\boldsymbol{w},
$$

where $u \equiv \boldsymbol{\psi}^{T} \boldsymbol{p}$ and $\boldsymbol{\psi}^{T} \boldsymbol{w}=0$. Multiplying both sides of (3.1) by $\boldsymbol{\psi}^{T}$ yields the equation

$$
\partial_{t} u=-\boldsymbol{\psi}^{T} \mathcal{L}\left(u \boldsymbol{p}^{s s}+\boldsymbol{w}\right) .
$$

Substitute $\boldsymbol{p}=u \boldsymbol{p}^{s s}+\boldsymbol{w}$ into 3.1 to get

$$
\partial_{t} u \boldsymbol{p}^{s s}+\partial_{t} \boldsymbol{w}=\frac{1}{\varepsilon} \widehat{A} \boldsymbol{w}-\mathcal{L}\left(u \boldsymbol{p}^{s s}+\boldsymbol{w}\right) .
$$

Using equation (3.3), this reduces to

$$
\partial_{t} \boldsymbol{w}=\frac{1}{\varepsilon} \widehat{A} \boldsymbol{w}-\left(I_{n}-\mathcal{P}\right) \mathcal{L}\left(u \boldsymbol{p}^{s s}+\boldsymbol{w}\right)
$$

where $I_{n}$ is the $n \times n$ identity matrix. Introduce a power series expansion for $\boldsymbol{w}$ with

$$
\boldsymbol{w} \sim \boldsymbol{w}_{0}+\varepsilon \boldsymbol{w}_{1}+\varepsilon^{2} \boldsymbol{w}_{2}+\ldots
$$

Substituting this into equation (3.5) and collecting terms $\mathcal{O}\left(\varepsilon^{-1}\right)$ yields the equation

$$
\widehat{A} \boldsymbol{w}_{0}=0 .
$$

Since $\boldsymbol{w}$ is orthogonal to the left nullspace of $\widehat{A}$, we have that $\boldsymbol{w}_{0}=0$. Collecting $\mathcal{O}(1)$ terms yields the equation

$$
\widehat{A} \boldsymbol{w}_{1}=\left(I_{n}-\mathcal{P}\right) \mathcal{L}\left(u \boldsymbol{p}^{s s}\right) .
$$

The orthogonal projection $I_{n}-\mathcal{P}$ ensures that the right-hand side of the above equation is in the range of $\widehat{A}$. Since $\widehat{A}$ is a singular matrix it follows from the Fredholm Alternative theorem that a family of solutions $\boldsymbol{w}_{1}$ exist. We can obtain a unique solution by requiring that $\boldsymbol{\psi}^{T} \boldsymbol{w}_{1}=0$.

It is convenient to define the mean of a vector $\mathbf{v}$ with respect to the stationary distribution $\boldsymbol{p}^{s s}$ according to

$$
\langle\mathrm{v}\rangle \equiv \mathbf{v}^{T} \boldsymbol{p}^{s s}
$$

Substituting $\boldsymbol{w} \sim \varepsilon \boldsymbol{w}_{1}$ into equation (3.3) yields

$$
\partial_{t} u=-\chi(x)\langle k\rangle u-\langle v\rangle \partial_{x} u+\langle B\rangle \partial_{x}^{2} u-\varepsilon \sum_{j=1}^{n} \mathcal{L}_{j} \partial_{x} w_{1, j} .
$$

From equation (3.8) we can see that the components of $\boldsymbol{w}_{1}$ are linear combinations of $u, \partial_{x} u$ and $\partial_{x}^{2} u$ so that we can write them as

$$
w_{1, j}=-q_{j} u-r_{j} \partial_{x} u-m_{j} \partial_{x}^{2} u
$$

where $q_{j}, r_{j}$ and $m_{j}, j=1, \ldots, n$, are $u$-independent. Collecting $\partial_{x} u$ terms in equation (3.8) yields an equation for $\boldsymbol{r}=\left(r_{1}, \ldots, r_{n}\right)^{T}$,

$$
\widehat{A} \boldsymbol{r}=-\left(\left(\langle v\rangle-v_{1}\right) p_{1}^{s s}, \cdots,\left(\langle v\rangle-v_{n}\right) p_{n}^{s s}\right)^{T} .
$$


The condition $\boldsymbol{\psi}^{T} \boldsymbol{w}_{1}=0$ implies that $\boldsymbol{\psi}^{T} \boldsymbol{r}=0$ and hence we can solve for $\boldsymbol{r}$ uniquely. Likewise equations for $\boldsymbol{q}$ and $\boldsymbol{m}$ are given by

$$
\begin{aligned}
& \widehat{A} \boldsymbol{q}=-\left(\left(\langle k\rangle-k_{1}\right) p_{1}^{s s}, \cdots,\left(\langle k\rangle-k_{n}\right) p_{n}^{s s}\right)^{T} \\
& \widehat{A} \boldsymbol{m}=-\left(\left(\langle B\rangle-B_{1}\right) p_{1}^{s s}, \cdots,\left(\langle B\rangle-B_{n}\right) p_{n}^{s s}\right)^{T} .
\end{aligned}
$$

Thus we obtain the Fokker-Plank equation

$$
\partial_{t} u=-\chi(x) \lambda u-V \partial_{x} u+D \partial_{x}^{2} u
$$

with

$$
\begin{aligned}
\lambda & =\langle k\rangle-\varepsilon \boldsymbol{k}^{T} \boldsymbol{q}+\mathcal{O}\left(\varepsilon^{2}\right) \\
V & =\langle v\rangle-\varepsilon \chi(x)\left(\boldsymbol{k}^{T} \boldsymbol{r}+\boldsymbol{v}^{T} \boldsymbol{q}\right)+\mathcal{O}\left(\varepsilon^{2}\right) \\
D & =\langle B\rangle+\varepsilon\left(\boldsymbol{v}^{T} \boldsymbol{r}+\chi(x)\left(\boldsymbol{k}^{T} \boldsymbol{m}+\boldsymbol{B}^{T} \boldsymbol{q}\right)\right)+\mathcal{O}\left(\varepsilon^{2}\right)
\end{aligned}
$$

Note that at $\mathcal{O}(\varepsilon)$ the drift velocity $V$ and effective diffusivity $D$ pick up additional terms within the target region in order to compensate for the flux into the target. In order to compute the $\mathcal{O}(\varepsilon)$ contributions to $V$ and $D$, the rank deficient equations (3.12)-(3.14) can be solved numerically using the full singular value decomposition of the matrix $\widehat{A}$ (for details see Appendix A). Note that if we neglect diffusion in the detached state so that $\boldsymbol{B}=0$ and $\boldsymbol{m}=0$, then equation (3.18) reduces to the simpler form

$$
D=\varepsilon \boldsymbol{v}^{T} \boldsymbol{r}+\mathcal{O}\left(\varepsilon^{2}\right) .
$$

The leading order contributions to $V$ and $D$ are then $x$-independent.

Initial conditions for the reduced FP equation (3.15) are chosen from the stationary distribution $\boldsymbol{p}^{s s}$ so that

$$
u(x, 0)=\delta(x-y) .
$$

Boundary conditions will be either

$$
\text { (reflecting) } V u-\left.D \partial_{x} u\right|_{x=0, L}=0,
$$

or

$$
\text { (periodic) } \quad V u-\left.D \partial_{x} u\right|_{x=0}=V u-\left.D \partial_{x} u\right|_{x=L} .
$$

Finally, it is useful to define the searching probability

$$
\sigma \equiv \sum_{j=1}^{n}\left(1-\delta_{k_{j}, 0}\right) p_{j}^{s s}
$$

to be the steady-state fraction of time spent in the searching phase. It follows that the steady-state fraction of time spent in the moving phase is $1-\sigma$. These two quantities are useful for characterizing intermittent behavior. With the definition of the target detection rates $k_{j}, j=1, \cdots, n$, given by $(2.16)$, we have that $\Lambda(x)=\chi(x) k_{0} \sigma+\mathcal{O}(\varepsilon)$.

To analyze the efficiency of the generalized intermittent search process we will use the FP equation (3.15) to calculate the MFPT $T$, which approximates the exact MFPT $\mathcal{T}$ defined by $(2.22)$. Let $\mathbb{P}(t)=\int_{0}^{L} u(x, t) d x$ be the total probability that the particle is still located in the domain $0<x<L$ at time $t$. Integrating equation (3.15) with respect to $x$ and using the reflecting boundary conditions (3.21), we have

$$
\frac{\partial \mathbb{P}}{\partial t}=-\lambda \int_{X-l}^{X+l} u(x, t) d x
$$


It follows that the total flux into the target is

$$
J(t)=\lambda \int_{X-l}^{X+l} u(x, t) d x
$$

The MFPT is then given by

$$
T=\int_{0}^{\infty} t J(t) d t
$$

There are two alternative methods for calculating $T$, one based on Laplace transforms and the other based on solving the corresponding backwards Fokker-Planck equation [38]. We will follow the former approach here. First, consider the Laplace transform of the probability flux $J$,

$$
\Upsilon(s) \equiv \int_{0}^{\infty} \mathrm{e}^{-s t} J(t) d t
$$

Taylor expanding the integral with respect to the Laplace variable $s$ shows that

$$
\begin{aligned}
\Upsilon(s) & =\int_{0}^{\infty} J(t)\left[1-s t+s^{2} t^{2} / 2-\ldots\right] \\
& =\left[1-s T+\frac{s^{2}}{2} T^{(2)}-\ldots\right],
\end{aligned}
$$

assuming that the moments

$$
T^{(n)}=\int_{0}^{\infty} t^{n} J(t) d t
$$

are finite. Thus, $\Upsilon(s)$ can be viewed as a generating function for the moments of the first passage time distribution [38]. Equations (3.25) and (3.27) imply that

$$
\Upsilon(s)=\lambda \int_{X-l}^{X+l} \widetilde{U}(x, s) d x,
$$

where $\widetilde{U}(x, s)$ is the Laplace transform of $u(x, t)$. Hence, we can proceed by solving the Laplace transformed Fokker-Planck equation to determine $\widetilde{U}(x, s)$. Substituting the result into equation (3.30) and Taylor expanding with respect to $s$ then allows us to extract $T$ using equation (3.28). In particular,

$$
T=-\left.\frac{d \Upsilon(s)}{d s}\right|_{s=0}=-\left.\lambda \int_{X-l}^{X+l} \frac{d \widetilde{U}(x, s)}{d s}\right|_{s=0} d x .
$$

\section{Optimal intermittent search and $[\mathrm{ATP}]$-dependence}

We can now use the QSS reduction to investigate how the efficiency of the random intermittent search process depends on various important biophysical parameters. Understanding such dependence provides insights into possible signaling mechanisms that could tune the efficiency of the intermittent search process. Here we focus on one particular control parameter, namely, the concentration $[\mathrm{ATP}]$ of intracellular ATP. At the single motor level, the stall force $F_{s}$, forward velocity $v_{f}$, and unbinding rate $\varepsilon_{0}$ all depend significantly on [ATP]. Hence, the velocities and state transitions of the full random intermittent search process will also depend on [ATP]. The QSS reduction provides a systematic procedure for calculating the effective diffusivity $D$, drift velocity $V$ and absorption rate $\Lambda$ of the FP equation (3.15) as functions of [ATP], and thus how the MFPT $T$ varies with [ATP]. We will consider both symmetric and asymmetric tug-of-war models and ignore the effects of diffusion in the search state by setting $D_{0}=0$. 


\subsection{Symmetric case}

The minimal model of intermittent search assumes unbiased motion [7], which significantly simplifies the calculation of the MFPT. Although motor-driven cargo transport is most often biased, it is possible for a motor-complex to exhibit unbiased motion when equal numbers of identical motors move on microtubules with opposite polarity, see Fig. 2. In this case the motor-complex is said to be symmetric. This assumption was also used in the original analysis of the tug-of-war model in terms of the stationary distribution [25]. Many experimental observations of bidirectional transport show strikingly similar statistics for transport in both directions so that on small enough time-scales unbiased motion is also physically justified. For continuity of our analysis with the previous development of both models, we start by considering the case of two identical sets of kinesin motors $\left(N_{+}=N_{-}=N\right)$ with opposite directional preference. When the motion is unbiased we have $V=0$ so that equation (3.15) simplifies to

$$
\partial_{t} u=-\lambda \chi(x) u+D \partial_{x}^{2} u
$$

with $\lambda$ and $D$ given by equations (3.16) and (3.19). In the symmetric case we can impose the reflecting boundary conditions

$$
\partial_{x} u(x, t)=0, \quad x=0, L .
$$

For simplicity we will also assume that the target is located in the center of the interval so that $X=L / 2$. We now calculate the MFPT $T$ by solving equation (4.1) in Laplace space, and then calculating the generating function $\Upsilon(s)$ using (3.30).

In order to handle the piecewise-constant function $\chi(x)$ we split the track up into three regions labelled $\rho=1,2,3$. Region two corresponds to the target interval $X-l \leq x \leq X+l$, whereas regions one and three are on either side of the target interval with $0 \leq x \leq X-l$ and $X+l \leq x \leq L$, respectively. We then partition the Laplace transformed solution $\widetilde{U}(x, s)$ in the three regions according to

$$
\begin{aligned}
& \widetilde{U}(x, s) \quad=\widetilde{U}_{1}(x, s), \quad 0 \leq x \leq L_{1} \equiv X-l \\
& \widetilde{U}(x+X-l, s)=\widetilde{U}_{2}(x, s), \quad 0 \leq x \leq L_{2} \equiv 2 l \\
& \widetilde{U}(x+X+l, s)=\widetilde{U}_{3}(x, s), \quad 0 \leq x \leq L_{3}=L-X-l .
\end{aligned}
$$

Setting the initial position of the cargo to be at location $y$ in region $\rho_{0}$, the Laplace transform of equation (4.1) can thus be written as

$$
D \partial_{x}^{2} \widetilde{U}_{\rho}-\left(\Lambda_{\rho}+s\right) \widetilde{U}_{\rho}=-\delta(x-y) \delta_{\rho, \rho_{0}} .
$$

where $\Lambda_{1,3}=0$, and $\Lambda_{2} \equiv \lambda$. The corresponding boundary conditions are

$$
\begin{array}{llrl}
\partial_{x} \widetilde{U}_{1}(0, s)=0, & & \widetilde{U}_{1}\left(L_{1}, s\right)=\Phi_{a}(s), \\
\widetilde{U}_{2}(0, s)=\Phi_{a}(s), & & \widetilde{U}_{2}\left(L_{2}, s\right)=\Phi_{b}(s), \\
\widetilde{U}_{3}(0, s)=\Phi_{b}(s), & & \partial_{x} \widetilde{U}_{3}\left(L_{3}, s\right)=0 .
\end{array}
$$

We have supplemented the external boundary conditions (4.2) with internal boundary conditions at either end of the target. The two unknown functions $\Phi_{a}(s)$ and $\Phi_{b}(s)$ can be determined self-consistently by imposing conservation of flux at the internal boundaries. This results in a $2 \times 2$ system of equations for $\Phi_{a}(s)$ and $\Phi_{b}(s)$. For ease

of notation, we suppress the explicit dependence of $\widetilde{U}_{\rho}$ and $\Phi_{a, b}$ on the initial location $x_{0}$ specified by the pair $\left(\rho_{0}, y\right)$. In terms of the original coordinate system,

$$
x_{0}=y \delta_{\rho_{0}, 1}+(y+X-l) \delta_{\rho_{0}, 2}+(y+X+l) \delta_{\rho_{0}, 3}
$$


Random intermittent search and the tug-of-war model of motor-driven transport 14

Solutions to equation (4.6) in each of the regions $0 \leq x \leq L_{\rho}, \rho=1,2,3$, are given by

$$
\begin{aligned}
\widetilde{U}_{1}(x) & =\delta_{\rho_{0}, 1} G_{1}(x, y)+\Phi_{a} F_{1}(x), \\
\widetilde{U}_{2}(x) & =\delta_{\rho_{0}, 2} G_{2}(x, y)+\Phi_{a} \widehat{F}_{2}(x)+\Phi_{b} F_{2}(x) \\
\widetilde{U}_{3}(x) & =\delta_{\rho_{0}, 3} G_{3}(x, y)+\Phi_{b} \widehat{F}_{3}(x)
\end{aligned}
$$

where we have now suppressed the explicit $s$-dependence, and

$$
\begin{aligned}
& F_{1}(x)=\frac{\cosh \left(\eta_{1} x\right)}{\cosh \left(\eta_{1} L_{1}\right)} \\
& \widehat{F}_{2}(x)=\frac{\sinh \left(\eta_{2}\left(L_{2}-x\right)\right)}{\sinh \left(\eta_{2} L_{2}\right)}, \quad F_{2}(x)=\frac{\sinh \left(\eta_{2} x\right)}{\sinh \left(\eta_{2} L_{2}\right)} \\
& \widehat{F}_{3}(x)=\frac{\cosh \left(\eta_{1}\left(L_{3}-x\right)\right)}{\cosh \left(\eta_{1} L_{3}\right)},
\end{aligned}
$$

with

$$
\eta_{1}=\sqrt{\frac{s}{D}}, \quad \eta_{2}=\sqrt{\frac{s+\lambda}{D}} .
$$

The initial condition is accounted for by the Green's functions $G_{\rho}(x, y)$ satisfying

$$
\left[D \partial_{x}^{2}-\left(s+\Lambda_{\rho}\right)\right] G_{\rho}(x, y)=-\delta(x-y)
$$

together with the homogeneous boundary conditions

$$
\begin{aligned}
& \partial_{x} G_{1}(0, y)=0, \quad G_{1}\left(L_{1}, y\right)=0, \\
& G_{2}(0, y) \quad=0, \quad G_{2}\left(L_{2}, y\right)=0 \text {, } \\
& G_{3}(0, y)=0, \quad \partial_{x} G_{3}\left(L_{3}, y\right)=0 .
\end{aligned}
$$

Thus,

$$
\begin{aligned}
& G_{1}(x, y)= \begin{cases}\frac{\cosh \left(\eta_{1} x\right) \sinh \left(\eta_{1}\left(L_{1}-y\right)\right)}{D \eta_{1} \cosh \left(L_{1} L_{1}\right)}, & x \in[0, y) \\
\frac{\left.\sinh \left(\eta_{1}\left(L_{1}-x\right)\right) \cosh \left(\eta_{1} y\right)\right)}{D \eta_{1} \cosh \left(\eta_{1} L_{1}\right)}, & x \in\left(y, L_{1}\right]\end{cases} \\
& G_{2}(x, y)= \begin{cases}\frac{\sinh \left(\eta_{2} x\right) \sinh \left(\eta_{2}\left(L_{2}-y\right)\right)}{D \eta_{2} \sinh \left(\eta_{2} L_{2}\right)}, & x \in[0, y) \\
\frac{\left.\sinh \left(\eta_{2}\left(L_{2}-x\right)\right) \sinh \left(\eta_{2} y\right)\right)}{D \eta_{2} \sinh \left(\eta_{2} L_{2}\right)}, & x \in\left(y, L_{2}\right]\end{cases} \\
& G_{3}(x, y)= \begin{cases}\frac{\sinh \left(\eta_{3} x\right) \cosh \left(\eta_{3}\left(L_{3}-y\right)\right)}{D \eta_{3} \cosh \left(\eta_{3} L_{3}\right)}, & x \in[0, y) \\
\frac{\cosh \left(\eta_{3}\left(L_{3}-x\right) \sinh \left(\eta_{3} y\right)\right)}{D \eta_{3} \cosh \left(\eta_{3} L_{3}\right)}, & x \in\left(y, L_{3}\right]\end{cases}
\end{aligned}
$$

Imposing conservation of flux at either end of the target yields a system of two equations for the two unknowns $\Phi_{a}, \Phi_{b}$.

$$
\begin{gathered}
\delta_{\rho_{0}, 2} \partial_{x} G_{2}(0, y)+\Phi_{a} \partial_{x} \widehat{F}_{2}(0)+\Phi_{b} \partial_{x} F_{2}(0) \\
=\delta_{\rho_{0}, 1} \partial_{x} G_{1}\left(L_{1}, y\right)+\Phi_{a} \partial_{x} F_{1}\left(L_{1}\right) \\
\delta_{\rho_{0}, 2} \partial_{x} G_{2}\left(L_{2}, y\right)+\Phi_{a} \partial_{x} \widehat{F}_{2}\left(L_{2}\right)+\Phi_{b} \partial_{x} F_{2}\left(L_{2}\right) \\
=\delta_{\rho_{0}, 3} \partial_{x} G_{3}(0, y)+\Phi_{b} \partial_{x} \widehat{F}_{3}(0)
\end{gathered}
$$

We introduce the following abbreviation for the various fluxes

$g_{\rho} \equiv \partial_{x} \widehat{F}_{\rho}\left(L_{\rho}\right), \quad h_{\rho} \equiv \partial_{x} F_{\rho}\left(L_{\rho}\right), \quad \bar{g}_{\rho} \equiv \partial_{x} F_{\rho}(0), \quad \bar{h}_{\rho} \equiv \partial_{x} \widehat{F}_{\rho}(0)$, 
and for the Green's functions

$$
\overline{\mathcal{G}}_{\rho}(y) \equiv \partial_{x} G_{\rho}(0, y), \quad \mathcal{G}_{\rho}(y) \equiv \partial_{x} G_{\rho}\left(L_{\rho}, y\right)
$$

Solving equation (4.26) for $\Phi_{b}$ yields

$$
\Phi_{b}\left(\rho_{0}, y\right)=\frac{1}{H_{2}}\left(\overline{\mathcal{G}}_{3}(y) \delta_{\rho_{0}, 3}-\mathcal{G}_{2}(y) \delta_{\rho_{0}, 2}-g_{2} \Phi_{a}\left(\rho_{0}, y\right)\right)
$$

where $H_{\rho}=h_{\rho}-\bar{h}_{\rho+1}$. Substituting equation (4.29) into equation (4.25) then yields

$\Phi_{a}\left(\rho_{0}, y\right)=\frac{H_{2}\left(\overline{\mathcal{G}}_{2}(y) \delta_{\rho_{0}, 2}-\mathcal{G}_{1}(y) \delta_{\rho_{0}, 1}\right)+\bar{g}_{2}\left(\overline{\mathcal{G}}_{3}(y) \delta_{\rho_{0}, 3}-\mathcal{G}_{2}(y) \delta_{\rho_{0}, 2}\right)}{H_{1} H_{2}+g_{2} \bar{g}_{2}}$

Combining equations (4.30), (4.29) and (4.12) yields the solution $\widetilde{U}_{2}$ which can then be substituted into equation (3.30) to obtain the generating function:

$$
\begin{aligned}
\Upsilon(s) & =\lambda \int_{0}^{2 l}\left(\delta_{\rho_{0}, 2} G_{2}(x, y)+\Phi_{a}\left(\rho_{0}, y\right) \widehat{F}_{2}(x)+\Phi_{b}\left(\rho_{0}, y\right) F_{2}(x)\right) d x \\
& =\lambda\left(\frac{-\sinh \left(\eta_{2} y\right)+\sinh \left(\eta_{2} L_{2}\right)+\sinh \left(\eta_{2}\left(y-L_{2}\right)\right)}{(s+\lambda) \sinh \left(\eta_{2} L_{2}\right)} \delta_{\rho_{0}, 2}+\frac{1}{\eta_{2}} \mathcal{X}_{\rho_{0}}(y) \tanh \left(\eta_{2} l\right)\right)
\end{aligned}
$$

where we have set $\mathcal{X}_{\rho_{0}}(y)=\Phi_{a}\left(\rho_{0}, y\right)+\Phi_{b}\left(\rho_{0}, y\right)$. Note that both $\eta_{1}$ and $\eta_{2}$ are functions of $s$, see equation (4.17). Finally, substituting equation (4.31) into (3.31) yields an analytical expression for the MFPT $\mathcal{T}\left(x_{0}\right)$, which depends on the initial location $x_{0}$ given by equation (4.10).

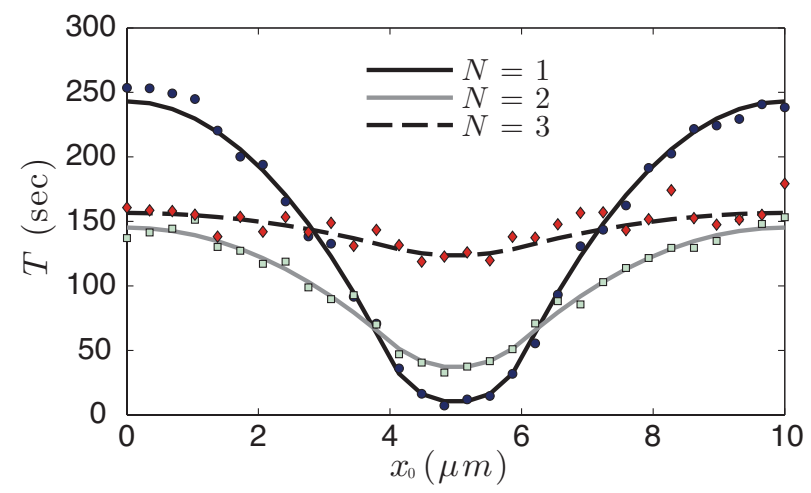

Figure 4. The MFPT $\mathcal{T}$ calculated using the QSS reduction (curves) is plotted along with averaged Monte-Carlo simulations (symbols) as a function of starting position $x_{0}$ for different numbers $N$ of kinesin motors. Parameter values are $l=1 \mu m, L=10 \mu m, X=5 \mu m, k_{0}=1 s^{-1}$, and $[\mathrm{ATP}]=100 \mu M$. Other biophysical parameter values are listed in Table 1. Monte-Carlo simulations were averaged over $(N+1)^{2} 10^{3}$ trials.

We now use our approximation of the MFPT based on the QSS reduction to explore how the efficiency of the search process depends on [ATP]. However, we first verify the accuracy of our QSS reduction by comparing the calculated $\mathcal{T}\left(x_{0}\right)$ to Monte-Carlo simulations of the full Markov process given by the CK equation (2.13), averaged over multiple runs. The results are shown in Fig. 4, which establish that there is excellent agreement between the full model and the reduced model in the chosen parameter regime. As expected the MFPT increases as the initial distance from the target increases. Recall that the target detection rate $\lambda$ is, to leading order, 

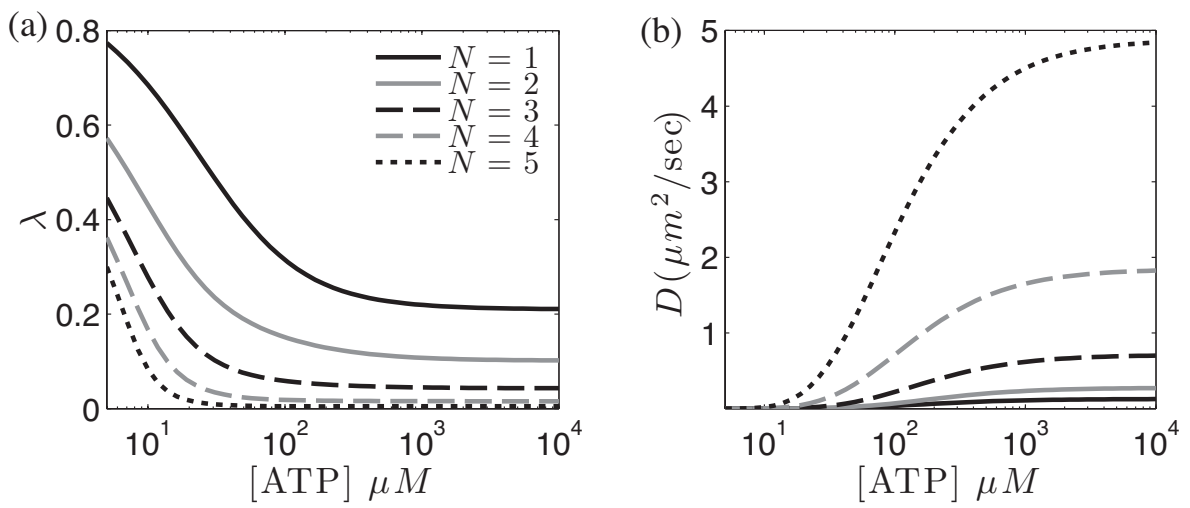

Figure 5. Results of the symmetric QSS reduction. (a) The effective target detection rate is plotted as a function of [ATP] for different numbers of motors $N$. This shows how [ATP] changes the behavior of the motor-complex by tuning the fraction of time it spends in the searching state. (b) The effective diffusion coefficient is plotted as a function of $[\mathrm{ATP}]$. Parameter values are the same as in Fig. 4.

the product of the base detection rate $k_{0}$ and the fraction of time spent searching (searching probability) defined by (3.23) so that $\lambda=k_{0} \sigma$. Since $k_{0}$ does not vary with $[$ ATP $]$ the target detection rate only changes due to the effect of $[$ ATP $]$ on the searching probability, as shown in Fig. 5(a). When ATP concentration is low the molecular motors have little fuel which weakens them so that the motor-complex is more likely to be in a searching state. As [ATP] increases the motors have sufficient fuel so that the complex spends more time moving and less time searching. However, since the motor-complex can't move while in a searching state, we also expect changes in [ATP] to have a strong effect on the mobility of the cargo so that increasing the searching probability will also slow the cargo down and increase the average delivery time. This can be seen in Fig. 5(b) where the diffusivity $D$, which determines the mobility of the motor-complex, increases with [ATP]. Thus, a large average delivery time can be the result of two factors: restricted mobility $(D \ll 1)$ at low [ATP], and a small searching probability $(\sigma \ll 1)$ at high $[\mathrm{ATP}]$. Balancing these two factors results in a minimum MFPT at a particular value of [ATP]. This is illustrated in Fig. 6, which plots the MFPT $\mathcal{T}$ averaged with respect to the starting position $x_{0}$ as a function of [ATP]. It can be seen that a sharp minimum in the MFPT emerges as the number of motors $N$ is increased.

\subsection{Asymmetric case}

In many examples of motor driven transport there are different types of molecular motors pulling the cargo in each direction, see Fig. 1. Directional asymmetry is established by the polarity of the microtubule. Kinesin motors pull cargo toward the $(+)$ end of the microtubule and dynein motors move toward the (-) end. Additionally, there may be an unequal number of $(+)$ end and $(-)$ end motors. This creates a bias in the direction the searching particle moves so that the mean velocity $\langle v\rangle$ is non-zero. Biased motor-transport is common among many cell types because cellular resources must be transported to specific regions within the cell. In terms of an intermittent 


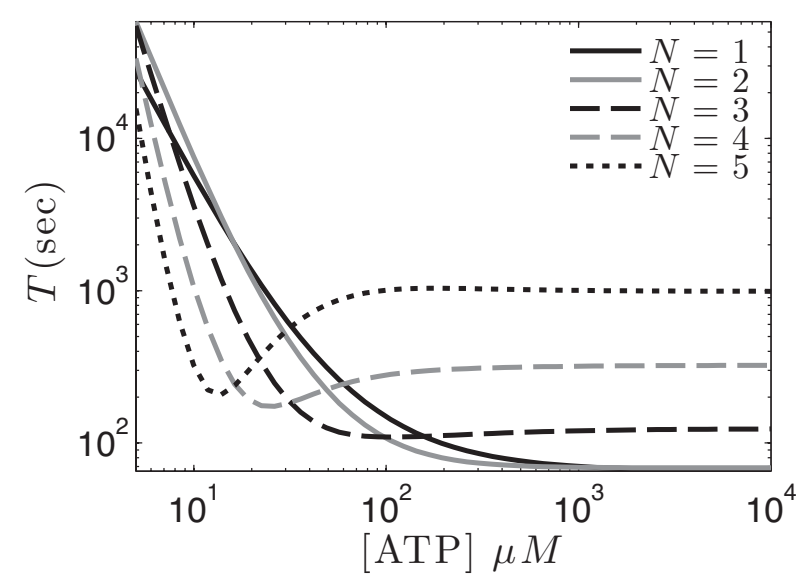

Figure 6. The MFPT $T\left(x_{0}\right)$ averaged with respect to the starting position $x_{0}$ is plotted as a function of [ATP]. The target is located at $X=5 \mu m$. Other parameter values are as in Fig. 4. A sharp minimum in the MFPT occurs as the number of motors is increased.

search, the goal is then to find the best search strategy when starting a distance away from target when the direction to the target (or targets) is initially known. This situation is different from the case of unbiased intermittent search where the searcher can start from anywhere within the track and the direction to the target is initially unknown. Therefore, here we assume that the search begins at the origin instead of averaging over the starting position as we did in the symmetric case. Finally, in order to formulate the biased intermittent search so that it is comparable to the symmetric case we use periodic boundary conditions so that the searcher finds a target with probability one.

Consider $N_{+}$forward moving kinesin motors and $N_{-}$backward moving dynein motors with $N_{+} \geq N_{-}$so that motion is biased in the forward direction. Note that because dynein is a slower and weaker motor than kinesin, motion will be biased even in the case where $N_{+}=N_{-}$. Following a similar procedure to the one used to calculate the generating function in the symmetric case we split the domain up into three regions labeled $\rho=1,2,3$. However, in the asymmetric case we must account for a non-zero drift velocity and periodic boundaries. Laplace transforming equation (3.15) with the initial condition $u_{\rho}(x, 0)=\delta(x) \delta_{\rho, 1}$ gives

$$
D \partial_{x}^{2} \widetilde{U}_{\rho}-V_{\rho} \partial_{x} \widetilde{U}_{\rho}-\left(\Lambda_{\rho}+s\right) \widetilde{U}_{\rho}=-\delta(x) \delta_{\rho, 1} .
$$

Here $D$ is given by equation (3.19), $\Lambda_{\rho}=\lambda \delta_{\rho, 2}$ with $\lambda=\langle k\rangle-\varepsilon \boldsymbol{k}^{T} \boldsymbol{q}$ and $V_{\rho}=$ $\langle v\rangle-\varepsilon \delta_{\rho, 2}\left(\boldsymbol{k}^{T} \boldsymbol{r}+\boldsymbol{v}^{T} \boldsymbol{q}\right)$. In each region we impose open boundary conditions with

$$
\begin{array}{ll}
\widetilde{U}_{1}(0, s)=\Phi_{p}(s), & \widetilde{U}_{1}\left(L_{1}, s\right)=\Phi_{a}(s), \\
\widetilde{U}_{2}(0, s)=\Phi_{a}(s), & \widetilde{U}_{2}\left(L_{2}, s\right)=\Phi_{b}(s),
\end{array}
$$

and

$$
\widetilde{U}_{3}(0, s)=\Phi_{b}(s), \quad \widetilde{U}_{3}\left(L_{3}, s\right)=\Phi_{p}(s) .
$$

The unknown functions $\Phi_{a}(s), \Phi_{b}(s)$, and $\Phi_{p}(s)$ will be determined by imposing conservation of flux at the boundaries between each region. For ease of notation we will suppress the explicit functional dependence on the Laplace variable $s$. 
Random intermittent search and the tug-of-war model of motor-driven transport 18

Consider the solution to the homogeneous $\mathrm{FP}$ equation that vanishes at $x=0$ :

$$
\psi_{\rho}(x)=\mathrm{e}^{\mu_{\rho+} x}-\mathrm{e}^{\mu_{\rho-} x}
$$

where

$$
\mu_{\rho, \pm}=\frac{V_{\rho}}{2 D} \pm \eta_{\rho}, \quad \eta_{\rho}=\sqrt{\left(\frac{V_{\rho}}{2 D}\right)^{2}+\frac{s+\Lambda_{\rho}}{D}} .
$$

This can be used to construct solutions to equation (4.32) in each region:

$$
\begin{aligned}
& \widetilde{U}_{1}(x)=G_{1}(x, 0)+\Phi_{p} \widehat{F}_{1}(x)+\Phi_{a} F_{1}(x) \\
& \widetilde{U}_{2}(x)=\Phi_{a} \widehat{F}_{2}(x)+\Phi_{b} F_{2}(x) \\
& \widetilde{U}_{3}(x)=\Phi_{b} \widehat{F}_{3}(x)+\Phi_{p} F_{3}(x) \\
& \widehat{F}_{\rho}(x)=\frac{\psi_{\rho}\left(x-L_{\rho}\right)}{\psi_{\rho}\left(-L_{\rho}\right)}, \quad F_{\rho}(x)=\frac{\psi_{\rho}(x)}{\psi_{\rho}\left(L_{\rho}\right)}
\end{aligned}
$$

The Green's function $G_{\rho}(x, y)$ satisfies open homogeneous boundary conditions so that

$$
G_{\rho}(x, y)= \begin{cases}\frac{\psi_{\rho}(x) \psi_{\rho}\left(y-L_{\rho}\right)}{D\left(\psi_{\rho}(y) \psi_{\rho}^{\prime}\left(y-L_{\rho}\right)-\psi_{\rho}^{\prime}(y) \psi_{\rho}\left(y-L_{\rho}\right)\right)}, & 0 \leq x<y \\ \frac{\left.\psi_{\rho}\left(x-L_{\rho}\right) \psi_{\rho}(y) \psi_{\rho}\left(y-L_{\rho}\right)\right)}{D\left(\psi_{\rho}(y) \psi_{\rho}^{\prime}\left(y-L_{\rho}\right)-\psi_{\rho}^{\prime}(y) \psi_{\rho}\right.}, & y<x \leq L_{\rho}\end{cases}
$$

Introducing the flux operator

$$
\mathcal{J}_{\rho}[f](x) \equiv V_{\rho} f(x)-D f^{\prime}(x),
$$

we impose conservation of flux at the three boundaries to yield a system of three equations for the three unknowns $\Phi_{p}, \Phi_{a}$, and $\Phi_{b}$ :

$$
\begin{gathered}
\Phi_{a} \mathcal{J}_{2}\left[\widehat{F}_{2}\right](0)+\Phi_{b} \mathcal{J}_{2}\left[F_{2}\right](0) \\
\quad=\Phi_{p} \mathcal{J}_{1}\left[\widehat{F}_{1}\right]\left(L_{1}\right)+\Phi_{a} \mathcal{J}_{1}\left[F_{1}\right]\left(L_{1}\right) \\
\Phi_{a} \mathcal{J}_{2}\left[\widehat{F}_{2}\right]\left(L_{2}\right)+\Phi_{b} \mathcal{J}_{2}\left[F_{2}\right]\left(L_{2}\right) \\
\quad=\Phi_{b} \mathcal{J}_{3}\left[\widehat{F}_{3}\right](0)+\Phi_{p} \mathcal{J}_{3}\left[F_{3}\right](0) \\
\mathcal{J}_{1}\left[G_{1}\right](0,0)+\Phi_{p} \mathcal{J}_{1}\left[\widehat{F}_{1}\right](0)+\Phi_{a} \mathcal{J}_{1}\left[F_{1}\right](0) \\
=\Phi_{b} \mathcal{J}_{3}\left[\widehat{F}_{3}\right]\left(L_{3}\right)+\Phi_{p} \mathcal{J}_{3}\left[F_{3}\right]\left(L_{3}\right)
\end{gathered}
$$

We introduce the following abbreviation for the various fluxes

$$
\begin{aligned}
& g_{\rho} \equiv \mathcal{J}_{\rho}\left[\widehat{F}_{\rho}\right]\left(L_{\rho}\right)=\frac{D \eta_{\rho} \mathrm{e}^{\frac{V_{\rho} L_{\rho}}{2 D_{\rho}}}}{\sinh \left(\eta_{\rho} L_{\rho}\right)} \\
& h_{\rho} \equiv \mathcal{J}_{\rho}\left[F_{\rho}\right]\left(L_{\rho}\right)=-D \eta_{\rho} \operatorname{coth}\left(\eta_{\rho} L_{\rho}\right)+\frac{V_{\rho}}{2} \\
& \bar{g}_{\rho} \equiv \mathcal{J}_{\rho}\left[F_{\rho}\right](0)=-\frac{D \eta_{\rho} \mathrm{e}^{\frac{-V_{\rho} L_{\rho}}{2 D_{\rho}}}}{\sinh \left(\eta_{\rho} L_{\rho}\right)} \\
& \bar{h}_{\rho} \equiv \mathcal{J}_{\rho}\left[\widehat{F}_{\rho}\right](0)=D \eta_{\rho} \operatorname{coth}\left(\eta_{\rho} L_{\rho}\right)+\frac{V_{\rho}}{2}
\end{aligned}
$$

For the Green's function we have $\mathcal{J}_{1}\left[G_{1}\right](0,0)=-1$. Using equation (4.46) to express $\Phi_{p}$ in terms of $\Phi_{a}$ and $\Phi_{b}$ and substituting the result into equations (4.44) and (4.45) gives

$$
\Phi_{a}=\frac{g_{1} \Delta_{3}+\bar{g}_{3} \Delta_{2}}{\Delta_{1} \Delta_{3}+\Delta_{2} \Delta_{4}}, \quad \Phi_{b}=\frac{-g_{1}}{\Delta_{3}}-\frac{\Delta_{4}}{\Delta_{3}} \Phi_{a}
$$


where

$$
\begin{array}{ll}
\Delta_{1}=\left(h_{3}-\bar{h}_{1}\right)\left(h_{1}-\bar{h}_{2}\right)+g_{1} \bar{g}_{1}, & \Delta_{2}=\left(h_{3}-\bar{h}_{1}\right) \bar{g}_{2}+g_{1} g_{3}, \\
\Delta_{3}=\left(h_{3}-\bar{h}_{1}\right)\left(h_{2}-\bar{h}_{3}\right)+g_{3} \bar{g}_{3}, & \Delta_{4}=\left(h_{3}-\bar{h}_{1}\right) g_{2}-\bar{g}_{1} \bar{g}_{3} .
\end{array}
$$

Substituting equation (4.52) into equation (4.39) then yields the solution in region 2 :

$$
\widetilde{U}_{2}(x)=\frac{g_{1} \Delta_{3}+\bar{g}_{3} \Delta_{2}}{\Delta_{1} \Delta_{3}+\Delta_{2} \Delta_{4}}\left(\widehat{F}_{2}(x)-\frac{\Delta_{4}}{\Delta_{3}} F_{2}(x)\right)-\frac{g_{1}}{\Delta_{3}} F_{2}(x) .
$$

Finally, we can compute the MFPT by substituting equation (4.54) into equation (3.31).
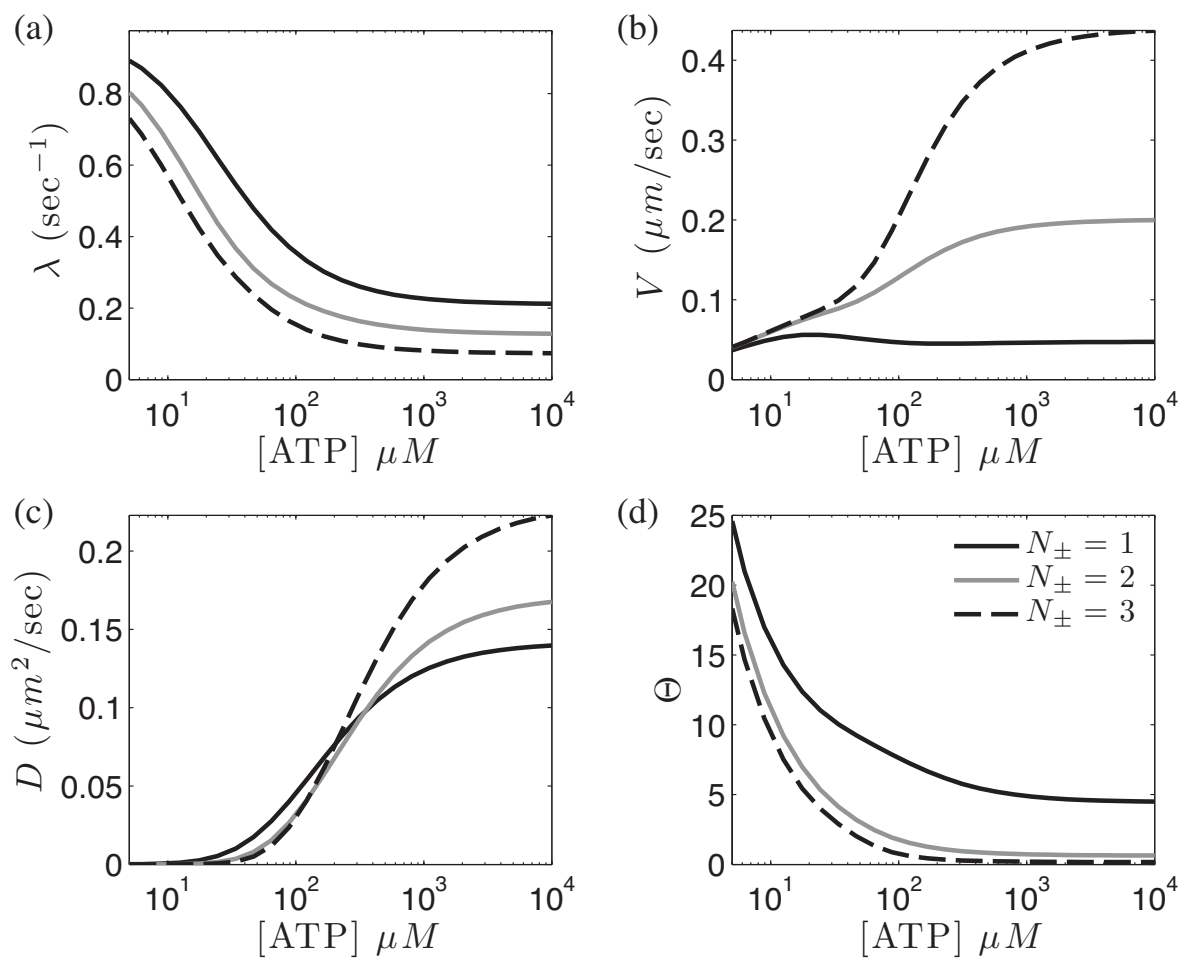

Figure 7. Results of the asymmetric QSS reduction. (a) The effective target detection rate $\lambda$ as a function of [ATP]. (b) The drift velocity $V$. (c) The effective diffusivity $D$. (d) The dimensionless detection efficiency $\Theta=l \lambda / V$. Parameter values are given by Table 1 and $k_{0}=1 s^{-1}$.

To illustrate how [ATP] affects the behavior of an asymmetric motor-complex we plot the detection rate $\lambda$, the drift velocity $V$ and the diffusivity $D$ as functions of [ATP] (Fig. 7). As in the symmetric case there are two competing factors that determine the average search time: detection efficiency and speed. The detection rate $\lambda$ is a decreasing function of [ATP] which means that the motor-complex searches more at low ATP concentration (Fig. 7(a)). Both $D$ and $V$ determine the mobility or speed of the motor complex and we find that they are both increasing functions of [ATP] (except for the case where $N_{+}=N_{-}=1$ where the motion of the motor-complex is only slightly biased. See Fig. 7(b,c)). The primary difference between the symmetric and asymmetric motor-complex is that for long time scales displacement from the 


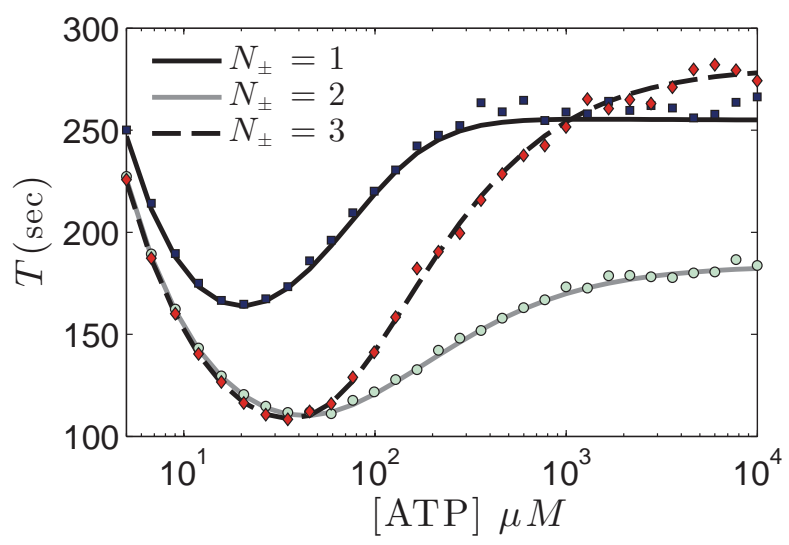

Figure 8. Results of the asymmetric ballistic search with $l=1 \mu m, X=10 \mu m$, $L=20 \mu m$ and $k_{0}=1 \mathrm{~s}^{-1}$. The MFPT $T$ (curves) is plotted along with averaged Monte-Carlo simulations (symbols) as a function of [ATP] for different numbers of kinesin motors $\left(N_{+}\right)$and dynein motors $\left(N_{-}\right.$. Monte-Carlo simulations we're averaged over $\left(N_{+}+1\right)\left(N_{-}+1\right) 10^{3}$ trials. A clear minimum in the MFPT suggests $[\mathrm{ATP}]$ is a useful control parameter for optimizing the search process.

starting position is dominated by the drift velocity $V$ rather than the diffusivity $D$. We can also define the dimensionless detection efficiency as $\Theta=\frac{l \lambda}{V}$ which simply represents the flux of probability into the target for a motor-complex moving with velocity $V$ over a distance $l$ (Fig. $7(\mathrm{~d})$ ). This quantifies the contribution a slower drift velocity adds to how well the motor-complex can detect the target. Thus, we can characterize detection with the parameter $\Theta$ which is maximized at low [ATP], and speed with the drift velocity $V$ which is maximized at high [ATP]. The result of the conflict between detection and speed is that the MFPT $\mathcal{T}$ is minimized at a particular value of $[$ ATP $]$ (Fig. 8).

In a biological system it may also be important for the minimum delivery time to be consistent for different numbers of motors. To see this we plot the minimum MFPT $T_{\min }$ for different numbers of kinesin and dynein motors (Fig. 9). In the symmetric case the minimum MFPT varied across the entire range of [ATP] as we changed the number of kinesin motors. When we change the number of kinesin and dynein motors in the asymmetric motor-complex the MFPT is minimized within a narrow band of [ATP] $(15-45 \mu M)$ that is consistent with levels found in vivo. We also see that $T_{\min }$ changes little $(110-160 s)$ suggesting that optimal mean target detection times are robust to changes in the motor complex.

\section{Discussion}

In this paper we have carried out a quasi-steady state analysis of a tug-of-war model of random intermittent search. After extending the tug-of-war model to include the location of the cargo we used a QSS reduction to calculate the MFPT to a hidden target as a function of the control parameter [ATP] and computed the MFPT for two cases: symmetric and asymmetric. In the symmetric case the motor-complex 


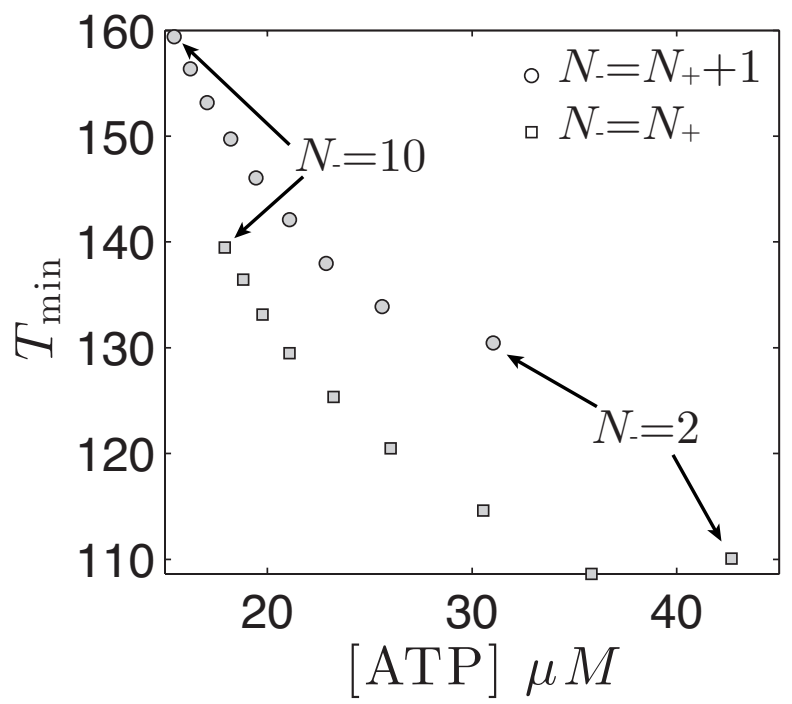

Figure 9. The minimum MFPT $T_{\min }$ as a function of [ATP] for different numbers $N_{ \pm}$of kinesin and dynein motors. The MFPT is minimized for all cases within a narrow band of $[\mathrm{ATP}]$. The variation in $T_{\min }$ for different numbers of motors is also small. This suggests that tuning [ATP] can also provide consistency in the delivery time independent of the particular choice of motor-complex. Parameter values are the same as in Fig. 8.

includes two identical populations of kinesin motors moving on two microtubules with opposite polarity so that the motion of the cargo is unbiased. We then computed the MFPT to the target when the search can start from anywhere within the track and the direction to the target is unknown. We also considered the more general case where the motor-complex contains a population of forward moving kinesin motors and backward moving dynein motors. The resulting motion of the motor-complex is biased and we computed the MFPT assuming that the search starts a distance away from the target and the direction to the target is initially known. In both the symmetric and asymmetric cases we found that [ATP] controls how much the cargo searches for the target. At low [ATP] the motor-complex spends most of its time searching and motion is slow, whereas at high [ATP] it rarely searches and motion is fast. Thus, the average delivery time can be optimized by properly tuning [ATP] to create a balance between speed and searching efficiency. Interestingly, when the motor-complex includes varying numbers of kinesin and dynein motors the MFPT is minimized within a small range of $[\mathrm{ATP}]$. The minimum MFPT was also found to vary little when we changed the number of motors, suggesting that optimizing motor dynamics is robust to the particular choice of how the motor-complex is constructed. These results lead us to conclude that the dynamic interactions between the motors pulling the cargo contribute to cargo localization. 
Random intermittent search and the tug-of-war model of motor-driven transport 22

\section{Appendix A. Solving the rank-deficient linear system for higher order terms}

To illustrate the procedure we will show how to compute the solution to the equation $L x=b$ also satisfying $\psi^{T} x=0$ provided that $b$ is in the range of $L$. Let $L=\mathrm{U} \Sigma \mathrm{V}^{T}$ be a full singular value decomposition (SVD) of $L$ where $\Sigma$ is a real diagonal matrix and $\mathrm{U}, \mathrm{V}$ are orthogonal matrices. It follows from our assumptions on $L$ that there is a single zero singular value $\sigma_{n}=0$. A normalized basis for the nullspace can be obtained from the $n$th column vector $\mathrm{v}_{n}$ of $\mathrm{V}$. The the stationary distribution $\boldsymbol{p}^{s s}$ is then given by

$$
\boldsymbol{p}^{s s}=\frac{\mathrm{v}_{n}}{\left\|\mathrm{v}_{n}\right\|_{1}} .
$$

Define the vectors $y=\mathrm{V}^{T} \mathrm{x}$ and $z=\mathrm{U}^{T} b$ so that $\Sigma y=z$. It follows that $y_{i}=z_{i} / \sigma_{i}$, $i=1, \cdots, n-1$. Since $\sigma_{n}=0$ the last element can be chosen arbitrarily to obtain a solution $y$. A standard least squares solution is obtained by setting $y_{n}=0$. However, we must determine $y_{n}$ using the condition $\sum_{i=1}^{n} \mathrm{x}_{i}=0$. Let $\mathrm{v}_{i, j}$ be the entry of $\mathrm{V}$ corresponding to the $i$ th column and $j$ th row. Since $\mathrm{x}=\mathrm{V} y$ we have

$$
\mathrm{x}_{i}=\sum_{j=1}^{n-1} \mathrm{v}_{i, j} y_{j}+\mathrm{v}_{i, n} y_{n} .
$$

Thus we require

$$
\sum_{i=1}^{n} \sum_{j=1}^{n-1} \mathrm{v}_{i, j} y_{j}+y_{n} \sum_{i=1}^{n} \mathrm{v}_{i, n}=0 .
$$

It follows that

$$
y_{n}=\frac{1}{\left\|\mathrm{v}_{n}\right\|_{1}} \sum_{i=1}^{n} \sum_{j=1}^{n-1} \mathrm{v}_{i, j} y_{j}
$$

The solution $\mathrm{x}$ is then given by

$$
\mathrm{x}=\mathrm{V} y \text {. }
$$

\section{Acknowledgements}

This publication was based on work supported in part by the NSF (DMS-0813677) and by Award No KUK-C1-013-4 made by King Abdullah University of Science and

Technology (KAUST). PCB was also partially supported by the Royal Society-Wolfson Foundation.

\section{References}

[1] Bell J W 1991 Searching behavior, the behavioral ecology of finding resources (London: Chapman and Hall)

[2] Viswanathan G, Buldyrev S, Havlin S, da Luz M, Raposo E and Stanley H. Optimizing the success of random searches, 1999 Nature 401 911-914

[3] Viswanathan G, Bartumeus F, Buldyrev S, Catalan J, Fulco U, Havlin S, da Luz M, Lyra M, Raposo E and Stanley H 2002 Physica A 314 208-213

[4] Berg O G, Winter R B and Von Hippel P H. Diffusion-driven mechanisms of protein translocation on nucleic acids. 1. Models and theory. 1981 Biochemistry 20 6929-6948 
[5] Halford S and Marko J. How do site-specific DNA-binding proteins find their targets? 2004 Nucleic Acids Res. 32 3040-3052

[6] Loverdo C, Benichou O, Moreau M and Voiturieez R. Enhanced reaction kinetics in biological cells 2008 Nat. Phys. 4 134-137

[7] Benichou O, Coppey M, Moreau M, Suet P, and Voituriez R, Optimal search strategies for hidden targets. Phys. Rev. Lett., 94:198101, 2005.

[8] Benichou O, Loverdo C, Moreau M, and Voituriez R, A minimal model of intermittent search in dimension two. J. Phys. A, 19: 065141, 2007.

[9] Oshanin O, Wio H S, Lindenberg K, and Burlatsky S Intermittent random walks for an optimal search strategy: one-dimensional case, 2007 J. Phys.: Condens. Matter 19065142.

[10] Loverdo C, Benichou O, Moreau M and Voituriez R Robustness of optimal intermittent search strategies in one, two, and three dimensions, 2009 Phys. Rev. E 80031146

[11] Oshanin O, Lindenberg K, Wio H S and Burlatsky S Efficient search by optimized intermittent random walks, 2009 J. Phys. A 42434008.

[12] Benichou O, Coppey M, Moreau M, Suet P and Voituriez R A stochastic model for intermittent search strategies, 2005 J. Phys.-Condes. Matter 17 S4275-S4286

[13] Pierce-Shimomura J, Morse T and Lockery S he fundamental role of pirouettes in C. elegans chemotaxis,1999 J. Neurosci. 19 9557-9569

[14] Huet S, Karatekin E, Tran V S, Fanget I, Cribier S and Henry J P Analysis of transient behavior in complex trajectories: application to secretory vesicle dynamics, 2006 Biophys. J. $913542-$ 3559

[15] Bressloff P C and Newby J Directed intermittent search for hidden targets, 2009 New J. Phys. 11023033

[16] Newby J M and Bressloff P C Directed intermittent search for a hidden target on a dendritic tree, 2009 Phys. Rev. E 80021913

[17] Knowles R B, Sabry J H, Martone M E, Deerinck T J, Ellisman M H, Bassell G J and Kosik K S Translocation of RNA granules in living neurons, 1996 J. Neurosci. 16 7812-7820

[18] Bannai H, Inoue T, Nakayama T, Hattori M and Mikoshiba K Kinesin dependent, rapid bidirectional transport of ER sub-compartment in dendrites of hippocampal neurons, 2004 J. Cell Sci. 117 163-175

[19] Gennerich A and Schild D Finite-particle tracking reveals submicroscopic-size changes of mitochondria during transport in mitral cell dendrites, 2006 Phys. Biol. 3 45-53

[20] Dynes J L and Steward O Dynamics of bidirectional transport of ARC MRNA in neuronal dendrites, 2007 J. Comp. Neurol. 500 433-447

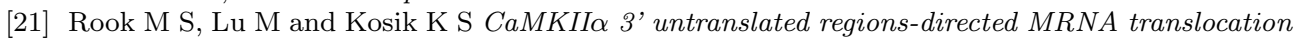
in living neurons: Visualization by GFP linkage, $2000 \mathrm{~J}$. Neurosci. 20 6385-6393

[22] Hirokawa N, Noda Y, Tanaka Y and Niwa S Kinesin superfamily motor proteins and intracellular transport, 2009 Nat Rev Mol Cell Biol 10 682-96

[23] Welte M Bidirectional transport along microtubules, 2004 Curr. Biol. 14 R525-R537

[24] Baas P W, Deitch J S, Black M M and Banker G A Polarity orientation of microtubules in hippocampal neurons: uniformity in the axon and nonuniformity in the dendrite, 1988 Proc. Natl. Acad. Sci. U. S. A. $858335-8339$

[25] Muller M J I, Klumpp S and Lipowsky R Tug-of-war as a cooperative mechanism for bidirectional cargo transport by molecular motors, 2008 Proc. Natl. Acad. Sci. U. S. A. 105 4609-4614

[26] Muller M J, Klumpp S and Lipowsky R Motility states of molecular motors engaged in a stochastic tug-of-war, 2008 J. Stat. Phys. 133 1059-1081

[27] Papanicolaou G and Kohler W Asymptotic theory of mixing stochastic ordinary di "erential equations, 1974 Commun. Pure Appl. Math. 27 641-668

[28] Gardiner C W 1983 Handbook of stochastic methods for physics, chemistry, and the natural sciences vol v. 13 (Berlin: Springer-Verlag) ISBN 0387113576 (U.S.)

[29] Visscher K, Schnitzer M and Block S Single kinesin molecules studied with a molecular force clamp, 1999 Nature 400 184-189

[30] Schnitzer M, Visscher K and Block S Force production by single kinesin motors, 2000 Nat. Cell Biol. 2 718-723

[31] King S J and Schroer T A Dynactin increases the processivity of the cytoplasmic dynein motor, 2000 Nat. Cell Biol. 2 20-24

[32] Gao Y Q A simple theoretical model explains dynein's response to load, 2006 Biophys. J. 90 $811-821$

[33] Ali Y M, Krementsova E B, Kennedy G G, Mahaffy R, Pollard T D, Trybus K M and Warshaw D M Myosin Va maneuvers through actin intersections and diffuses along microtubules, 2007 Proc. Natl. Acad. Sci. U. S. A. 104 4332-4336 
Random intermittent search and the tug-of-war model of motor-driven transport 24

[34] Ross J L, Ali M Y and Warshaw D M Cargo transport: molecular motors navigate a complex cytoskeleton, 2008 Curr. Opin. Cell Biol. 20 41-47

[35] Ali Y M, Lu H, Bookwalter C S, Warshaw D M and Trybus K M Myosin V and Kinesin act as tethers to enhance each others' processivity, 2008 Proc. Natl. Acad. Sci. U. S. A. 105 4691-4696

[36] Berger F, Mueller M J I and Lipowsky R Enhancement of the processivity of kinesin-transported cargo by myosin V, 2009 Epl 8728002.

[37] Posta F, D'Orsogna M R and Chou T Enhancement of cargo processivity by cooperating molecular motors, 2009 Phys. Chem. Chem. Phys. 11 4851-4860

[38] Redner S 2001 A guide to first-passage processes (Cambridge, UK: Cambridge University Press) ISBN 0521652480 



\section{RECENT REPORTS}

28/09 Quasi-steady state reduction of molecular motor-based models of directed intermittent search

Newby

Bressloff

29/09 All-at-once preconditioning in PDE-constrained optimization Rees

Stoll

Wathen

30/09 An hp-Local Discontinuous Galerkin method for Parabolic Pani Integro-Differential Equations

Yadav

31/09 Stochastic neural field theory and the system-size expansion

Bressloff

32/09 A Hamiltonian Krylov-Schur-type method based on the symplectic Lanczos process

Benner

Faßbender

Stoll

33/09 Nematic liquid crystals : from Maier-Saupe to a continuum theory

Ball

Majumdar

34/09 Tangent unit-vector fields: nonabelian homotopy invariants and the Dirichlet energy

Majumdar

Robbins

Zyskin

35/09 A metabolite-sensitive, thermodynamically-constrained model of

Tran cardiac cross-bridge cycling: Implications for force development during ischemia

Smith

Loiselle

Crampin

36/09 Modelling bacterial behaviour close to a no-slip plane boundary: the influence of bacterial geometry

Shum

Gaffney

Smith

37/09 Optimal L2-error estimates for the semidiscrete Galerkin approx-

Goswami imation to a second order linear parabolic initial and boundary value problem with nonsmooth initial data

38/09 Optimal L2 estimates for semidiscrete Galerkin methods for parabolic integro-differential equations with nonsmooth data

Pani

Goswami

Pani

Yadav

39/09 Spatially structured oscillations in a two-dimensional excitatory neuronal network with synaptic depression

Kilpatrick

Bressloff

40/09 Stationary bumps in a piecewise smooth neural field model with synaptic depression

Kilpatrick

Bressloff

41/09 Homogenization for advection-diffusion in a perforated domain Haynes

Hoang

Norris

Zygalakis 
42/09 Fast stochastic simulation of biochemical reaction systems by alternative formulations of the Chemical Langevin Equation

Melykuti

Burrage

Zygalakis

43/09 Pseudoreplication invalidates the results of many neuroscientific

Lazic studies

44/09 Cardiac cell modelling: Observations from the heart of the cardiac physiome project

45/09 A Hybrid Radial Basis Function - Pseudospectral Method for Thermal Convection in a 3-D Spherical Shell

Wright

Flyer

46/09 Refining self-propelled particle models for collective behaviour

Yates

Baker

Erban

Maini

47/09 Stochastic Partial Differential Equations as priors in ensemble methods for solving inverse problems

Potsepaev

Farmer

Aziz

48/09 DifFUZZY: A fuzzy spectral clustering algorithm for complex data

Cominetti et al. sets

01/10 Fluctuations and instability in sedimentation

Guazzelli

Hinch

02/10 Determining the equation of state of highly plasticised metals from Hinch boundary velocimetry

03/10 Stability of bumps in piecewise smooth neural

Kilpatrick elds with nonlinear adaptation

Bressloff

Copies of these, and any other OCCAM reports can be obtained from:

Oxford Centre for Collaborative Applied Mathematics Mathematical Institute

24 - 29 St Giles'

Oxford

OX1 3LB

England

www.maths.ox.ac.uk/occam 